\title{
Haemonchus contortus e Medidas Estratégicas de Controle para Ovinos
}

\author{
Haemonchus contortus and Strategic Control Measures for Sheep
}

\author{
Janaína Palermo Mendes*a; Talize Tieme Tsuzukib; Marcos Barbosa Ferreirac ${ }^{\mathrm{c}}$; Wagner Rodrigues Garcia \\ Jean Kaique Valentima; Rita Therezinha Rolim Pietramale ${ }^{\mathrm{a}}$ \\ ${ }^{a}$ Universidade Federal da Grande Dourados, Programa de Pós-Graduação Stricto Sensu em Zootecnia. MS, Brasil. \\ bUniversidade Anhanguera Uniderp. MS, Brasil. \\ 'Universidade Anhanguera - Uniderp, Programa de Pós-Graduação Stricto Sensu em Produção e Gestão Agroindustrial. MS, Brasil. \\ *E-mail: janapalermo@gmail.com
}

\begin{abstract}
Resumo
Ovinos são comumente acometidos por parasitoses gastrointestinais, a maioria cosmopolita, sendo Haemonchus sp., o nematódeo encontrado com maior frequência parasitando os ovinos, que se contaminam ingerindo as larvas. Isso implica na importância de se conhecer o ciclo de vida deste parasito, que é um micro-organismo microscópico, que se aloja no trato gastrointestinal e absorve o sangue, ocasionando o quadro de anemia, fato este que pode debilitar rapidamente o animal e causar o óbito, além de provocar um atraso no desenvolvimento do animal, gerando perdas econômicas. Foi utilizada, neste estudo, a metodologia da revisão bibliográfica, procedente de artigos científicos e livros. O H. contortus é a espécie de maior relevância, pois tem alta patogenicidade e alta prevalência nos rebanhos brasileiros, causando perdas econômicas ao produtor em função da baixa produtividade e desenvolvimento dos animais acometidos, como perda de peso, anemia, atraso no desenvolvimento corporal de animais jovens e, causando o óbito. O uso indiscriminado de antiparasitários causou o aumento da resistência dos helmintos às moléculas existentes no mercado. Dessa forma, a necessidade de buscar novos métodos de controle de verminoses visa diminuir o uso de anti-helmínticos por meio da vermifugação seletiva, alimentação balanceada com suplementação proteica e o fornecimento de forrageiras de boa qualidade e com altos teores de proteína.
\end{abstract}

Palavras-chave: Haemonchus contortus. Ovinos Pantaneiros. Prevalência. Resistência Parasitária.

\begin{abstract}
Sheep are commonly affected by gastrointestinal parasites, most of them cosmopolitan, Haemonchus sp., a nematode most commonly found parasitizing sheep, whichare contaminated when they ingest the larvae. This implies the importance of knowing the life cycle of this parasite, which is a microscopic microorganism that lodges in gastrointestinal tract and absorbs blood and causes anemia, a fact that can quickly weaken the animal and cause death, in addition to causing a delay in the animal's development, generating economic reduction. It was used in this study, the literature review methodology, originated from scientific articles and books. H. contortus is a species of greater relevance because it has high pathogenicity and high prevalence in Brazilian herds, economic damage and due to the low developmental and developmental rates of the affected animals, such as weight loss, anemia, delayed body development of young animals and cause death. The indiscriminate use of antiparasitics causes increased helminths resistance to molecules existing in the market. Therefore, it is necessary to search for new worms control methods that reduce the use of anthelmintics through selective deworming, feeding in a balanced way with protection supplements and supplying good quality forages with high levels of protein.
\end{abstract}

Keywords: Haemonchus contortus. Pantaneiro Sheep. Prevalence. Parasitic Resistance.

\section{Introdução}

A ovinocultura brasileira se encontra em expansão, pois atualmente há uma maior demanda do mercado consumidor por produtos de melhor qualidade. O rebanho ovino brasileiro está estimado em 17.976,367 cabeças e no Município de Campo Grande/MS se estima uma população de 15.165 cabeças (IBGE, 2018) entre raças com aptidões para carne, leite, pele e lã.

Em decorrência a situação atual produtiva, representada por uma pequena parcela na produção de carne in natura, a baixa produtividade, o manejo inadequado e pouca tecnologia são fatores desafiadores para a produção eficiente dos ovinos. Nesse contexto, depara-se com um dos maiores problemas dos criadores de ovinos, os parasitos gastrointestinais, principalmente eimerioses e helmintoses, que representa o maior e mais grave problema sanitário, e gera relevantes perdas inviabilizando economicamente a criação (VIEIRA, 2007).

Tais perdas ocorrem em virtude da baixa produtividade de animais acometidos pelo Haemonchus contortus com $\mathrm{o}$ alto índice de mortalidade, alta morbidade e atraso no desenvolvimento corporal de animais jovens (CHAGAS et al. 2005) decorrente principalmente da dificuldade do controle dos parasitas, seja por informações inadequadas ou tratamentos sem eficácia (CLIMENI et al. 2008).

A presente revisão tem como objetivo revisar e descrever a importância do parasito gastrointestinal $H$. contortus para criação do rebanho de ovinos do grupo genético pantaneiro em função de sua alta prevalência, além de medidas para o controle estratégico de parasitoses. 


\section{Desenvolvimento}

\subsection{Metodologia}

Esta revisão bibliográfica foi fundamentada por meio de artigos científicos, livros e dissertações disponíveis nos meios de buscas como Google Acadêmico, Elsevier, Mendeley, Science Direct, Scielo, Endnote. Estas ferramentas fornecem bases de dados significativos sobre a literatura pesquisada, sendo esses de revistas científicas e de publicações relevantes. Foi empregado na busca, o uso das seguintes palavras-chaves, parasitas gastrointestinais, Haemonchus contortus, ovinos, parasitose em ovinos.

O método de investigação consiste em analisar os conteúdos de artigos em publicações que se relacionam com H. contortus e medidas estratégicas para o controle deste parasita em ovinos. Foram selecionados dezoito trabalhos científicos publicados nos últimos dez anos.

\subsection{Haemonchus contortus}

H. contortus é um helminto hematófago, pertence ao filo Nemathelminthes, classe Nematoda, família Trichostrongylidae, subfamília Haemoncinae e gênero Haemonchus (ENDO et al., 2014), que causa a perda de eritrócitos e ferro, levando à inflamação da mucosa abomasal e ativação do sistema imune (SCZESNY-MORAES et al. 2010; COSTA et al. 2011; STORILLO, 2016). As larvas e parasitos adultos no abomaso causam o aumento do $\mathrm{pH}$ e gastrite, comprometendo a capacidade digestória, ocasionando um quadro de perda energética e proteica e anorexia (MILLER et al. 2006). O ciclo biológico é direto e se inicia com parasitos adultos presentes no abomaso do hospedeiro, tendo estes parasitos alta capacidade de oviposição e os ovos morulados são eliminados nas fezes. A fase ambiental se inicia no bolo fecal, em presença de oxigênio, os ovos eclodem em larvas de primeiro estágio (L1), alimentando-se nesse ambiente e faz a muda para larva de segundo estágio (L2) e, posteriormente, em larva de terceiro estágio (L3), que é infectante, não se alimenta, pois mantem a cutícula do estágio anterior. Esta parte do ciclo ocorre em condições ideais de temperatura entre 18 a $26{ }^{\circ} \mathrm{C}$ e umidade entre 80 a $100 \%$ (CLIMENI et al. 2008; AMARANTE, 2014; STORILLO, 2016). As L3 migram erraticamente para fora do bolo fecal, auxiliados pela presença de umidade até o capim, dessa forma são ingeridas pelo hospedeiro (AMARANTE, 2014).

De acordo com Melo et al. (2003), após a ingestão, no estabelecimento inicial da hemoncose, ocorre o turnover dos parasitos adultos, ou seja, há um controle natural da carga parasitária pela relação de quantidade de larvas ingeridas e a mortalidade dos adultos, resultando no equilíbrio dinâmico entre a taxa de ingestão de larvas, taxa de mortalidade e reposição. Alguns triconstrogilídeos não têm a população parasitária regulada por esse mesmo processo (MELO et al. 2003). Os parasitos adultos podem ser identificados macroscopicamente em função do local específico, o abomaso, e o tamanho que varia de 1,1 a 2,7 cm de comprimento (ENDO et al. 2014).

Segundo Vieira (2007), os efeitos do parasitismo no rebanho se manifestam de várias formas, conforme as espécies presentes, a intensidade da infecção, a categoria e o estado fisiológico e nutricional do hospedeiro. O diagnóstico, de forma geral, é baseado em sinais clínicos, que na forma aguda apresentam perda de peso, diarreia, pelos arrepiados e sem brilho, anemia, desidratação e edema submandibular (FONSECA et al., 2011). A coprologia ajuda identificar a presença de ovos e larvas nas fezes (ROBERTO, 2018) e os achados na necropsia para verificar as lesões, assim como contagem de parasitos presentes no abomaso e outras espécies que possam acometer o hospedeiro (SCZESNY-MORAES et al., 2010).

\subsection{Epidemiologia}

A criação de ovinos no Brasil ainda é de forma extensiva, ocasionando prevalência de animais acometidos no rebanho, já que não há manejo adequado de pastagens, separação de lotes de acordo com a idade, além de inadequada verificação clínica preventiva. Desse modo, a pastagem constitui um importante veículo de transmissão oral dos parasitos gastrointestinais (BRAGA; GIRARDI, 1991; SPRENGER et al. 2012).

Os helmintos não se distribuem de maneira uniforme em um rebanho ovino, mesmo que os animais sejam de mesma raça e idade (NIETO et al. 2003; STEAR; MURRAY, 1994). No entanto, segundo Girão et al. (1980), a ocorrência de verminose em animais jovens é maior quando comparada aos adultos que possuem imunidade adquirida por infecções ocorridas anteriormente (AHID et al. 2008), sendo estes a principal fonte de infecção para ovinos jovens. H. contortus acomete também os bovinos, sendo patogênico para bezerros (AMARANTE, 2014; FÁVERO; STORILLO, 2016).

Para Vieira (2007), a precipitação pluviométrica é o fator climático mais importante no aparecimento de infecções por nematoides gastrointestinais em rebanhos, sendo um aspecto fundamental, visto que a transmissão da maioria dos nematódeos só ocorre com precipitação mínima mensal de $50 \mathrm{~mm}$.

Na região Nordeste, as áreas de criação extensiva nas quais os ovinos e os caprinos são mantidos é exclusivamente na caatinga, as infecções por parasitos gastrointestinais são raras, quando postos com baixas lotações de um a dois animais por hectare. Com a adoção de técnicas semi-intensivas de produção há maior susceptibilidade para as infecções (COSTA et al. 2011).

O uso de pastagens cultivadas, que proporcionam sombreamento e evitam a dessecação de ovos e larvas, criaram condições ideais de sobrevivência dos estádios larvais e ovos dos parasitos no ambiente (COSTA et al. 2011). Dessa forma, há aumento significativo na frequência de helmintoses gastrointestinais no rebanho nessas áreas para pastejo. 
Pastagens irrigadas podem influenciar na disponibilidade de L3, sendo encontradas larvas maciçamente no verão e em temperatura de $24^{\circ} \mathrm{C}$ (FONSECA et al. 2011). Temperatura e umidade são fatores determinantes na sobrevivência das larvas em vida livre.

Outro fator que influencia a vida livre de nematoides gastrointestinais no ambiente são as características morfofisiológicas das plantas forrageiras como a estrutura foliar, hábito de crescimento e produção de massa verde, associados aos fatores climáticos (ROBERTO, 2018). As forrageiras que proporcionam a incidência de raios solares no estrato inferior das touceiras e solo propiciam sombreamento e umidade podendo potencializar a reinfecção dos animais. Dessa forma, a avaliação de espécies forrageiras se torna uma alternativa importante no controle de nematoides gastrointestinais (LOPES et al. 2016).

\subsection{Prevalência}

Gonçalves e Vieira (1963) já haviam confirmado que $H$. contortus é o nematódeo mais nocivo a ovinos. Braga e Girardi (1991) constataram que larvas de $H$. contortus apareceram durante o ano todo do período experimental, demonstrando ser esta espécie mais prevalente em relação às outras encontradas.

Sotomaior e Thomaz-Soccol (2001), ao avaliarem a prevalência de $H$. contortus em ovinos, observaram 83,2\% de presença de ovos dos parasitos nas fezes. Seguindo a mesma linha de pesquisa, Ramos et al. (2004) e Amarante (2005), ao promoverem o estudo epidemiológico de helmintos gastrintestinais de ovinos em Botucatu, relataram a presença de $100 \%$ de $H$. contortus, Brito et al. (2009) obtiveram o resultado de $63,54 \%$ em um estudo realizado em uma microrregião do Estado do Maranhão e outro realizado no Mato Grosso do Sul por Sczesny-Moraes et al. (2010), com $73,4 \%$ em necropsias de animais adultos. Apenas Ahid et al. (2007) encontraram resultados inferiores com 22,6\% para Haemonchus sp. em análises que aconteceram no Rio Grande do Norte.

\subsection{Controle estratégico}

O uso do controle estratégico de parasitas é necessário, pois reduz a contaminação dos animais e do ambiente. No Brasil foram revisadas algumas técnicas como o manejo do rebanho e pastagens, descontaminação prévia da pastagem com pastoreio rotacionado e/ou pastoreio com alternância de espécie e de categorias de hospedeiros, nutrição, seleção genética, controle biológico, vacinas, fitoterapia (CEZAR et al. 2008).

Conforme observações de Gonçalves e Vieira (1963), Braga e Girardi (1991), Vieira (2007), os controles estratégicos devem se concentrar no período de maior deficiência hídrica, pois o tratamento reduz o número de helmintos nesse período, sendo desfavorável para as formas de vida livre. Corroborando com as informações de Da Silva et al. (2017), que afirmam que tais parasitas são sensíveis ao clima e sua sazonalidade.

O método Famacha ${ }^{\circledR}$ tem sido conhecido como um tipo de tratamento seletivo, uma vez que apenas animais acometidos e que apresentem anemia clínica são destinados à vermifugação (MONTEIRO et al. 2017), sendo assim um dos mais utilizados no controle de parasitas em pequenos ruminantes, segundo Fernandes et al. (2015). Essa técnica consiste em avaliar a mucosa, definida a partir da coloração da conjuntiva, determinando o grau de anemia dos animais, conforme as categorias que variam de 1 a 5 , sendo 1 uma coloração vermelha brilhante e 5 sendo coloração pálida, quase branco, representando os diferentes valores de hematócrito (BATH; VAN WYK, 2001).

Porém, apesar do uso generalizado, este método deve ser utilizado juntamente com outras técnicas, visando o melhor controle da população de parasitos presente no rebanho (AMADUCCI et al. 2017), evitando assim uso prolongado ou inadequado de um mesmo princípio ativo para o controle eficiente dessas parasitoses e a resistência parasitária, buscando uma produção animal economicamente mais eficiente com redução de custos em tratamentos.

A contagem do número de ovos por grama de fezes (OPG) deve ser utilizada como apoio ao controle estratégico para redução da carga parasitária, especialmente na seca, quando as fases imaturas estarão susceptíveis à destruição por dessecação no ambiente e uso de anti-helmínticos em doses adequadas (AHID et al. 2007) e com ajuda do método Famacha ${ }^{\circledR}$, recomenda-se medicar o menor número de animais possível e com menor frequência (FERNANDES et al. 2015).

Bath e Van Wyk (2001) utilizaram o método Famacha ${ }^{\circledR}$ em diferentes regiões da África do Sul, no período de 1998 a 1999 avaliando dez rebanhos. Estes autores observaram uma redução entre 38 e 96\%, com média de 58,4\% na utilização e nos custos com dosificações. No Brasil, Molento e Dantas (2001) avaliaram, em dados preliminares, que após a utilização deste método no período de 120 dias (março a junho de 2000), foi possível reduzir em 79,5\% as aplicações de antiparasitários.

Em experimento realizado por Reis (2004) seconstatou que $H$. contortus foi o parasito mais frequente ao início do experimento e, após o período de um ano, houve predominância de Trichostrongylus spp., não sendo este controlado pelo método Famacha ${ }^{\circledR}$.

Praticamente todas as moléculas anti-helmínticas existentes no Brasil são ineficazes para controle de helmintíases por conta de resistência adquirida ao longo dos anos. Em 2012, houve o lançamento brasileiro da nova molécula Monepantel (Zolvix ${ }^{\circledR}$, monepantel 2,5\%, Novartis Saúde Animal) para ovinos e caprinos, sendo relatada eficácia de $100 \%$ de ação anti-helmíntica. Kaminsky et al. (2011) relataram 100\% de eficácia do monepantel em dois grupos de estudo, Baker et al. (2012) obtiveram resultados parecidos. Em contrapartida, a resistência ao Monepantel foi relatada primeiramente na Nova Zelândia, em 2013, em nematoides Teladorsagia 
circumcincta e Trichostrongylus colubriformis. Desde então foi relatada a resistência também por $H$. contortus no Uruguai (MEDEROS et al., 2014), na Holanda (VAN DEN BROM et al. 2015), Austrália (SALES; LOVE, 2016, EMERY et al., 2016) e no Brasil por De Albuquerque et al. (2017) e Martins et al. (2017).

Conforme observado a campo pelos autores da presente revisão, o monepantel quando usado maciçamente e com intervalos inferiores ao recomendado é indicativo para a causa da resistência ao princípio ativo, outras causas de resistência podem ser relatadas como subdoses e escolha errada do vermífugo. $\mathrm{O}$ uso do anti-helmíntico monepantel em dosagem de $1 \mathrm{ml} / 10 \mathrm{~kg}$ tem surtido efeito positivo e eficaz no controle de parasitoses gastrointestinais em ovinos e como tratamento coadjuvante em função do estado anêmico que os animais se encontram, sendo utilizado o uso de um complexo vitamínico B12 injetável. A anemia causada por $H$. contortus associada à deficiência de Vitamina B12 e cobalto leva a um distúrbio do metabolismo do ácido propiônico acompanhado da perda de apetite e morte por inanição (RADOSTITIS et al. 2002).

Conforme Costa et al. (2011), existem outras formas de tratamento com anti-helmínticos como o tratamento preventivo, curativo, tático, supressivo e não intencional. O tratamento preventivo consiste no uso de anti-helmíntico em todo o rebanho, em períodos regulares e datas preestabelecidas com objetivo de evitar infecções clínicas e subclínicas. Já o tratamento curativo visa tratar somente animais acometidos com sinais clínicos evidentes.

O uso do tratamento tático é realizado sempre que as condições ambientais favorecem o surgimento da verminose, como períodos de menor precipitação hídrica (COSTA et al., 2011), o mesmo ocorre no supressivo, que preconiza vermifugar os animais a cada 2-4 semanas, com drogas de curta persistência e antes do período pré-patente dos parasitas, objetivando a quase total eliminação no ambiente (REIS, 2004; TORRES-ACOSTA; HOSTE, 2008), e o tratamento não intencional que é quando se utiliza o anti-helmíntico para tratamento de outras doenças parasitárias como Oestrus ovis, Dermatobia hominis e sarna.

A introdução de boa nutrição energética e proteica aumenta resistência dos ovinos para as infecções e a correta suplementação do rebanho leva à diminuição do grau de infecção por parasitos (COSTA et al. 2011). Já as carências alimentares por determinados períodos aumentam a susceptibilidade às infecções e favorecem a ocorrência de sinais clínicos (TORRES-ACOSTA; HOSTE, 2008).

O confinamento se torna um sistema eficiente para eliminação das infecções por helmintos gastrointestinais com pastagens cortadas de locais sem pastoreio, água limpa e potável e com a devida higienização do local. As larvas L3 não sobrevivem no feno e na silagem (TORRES-ACOSTA; HOSTE, 2008) em função de baixa concentração hídrica e pH ácido (VELHO et al. 2007).

O manejo integrado é eficaz quando realizado corretamente, como limpeza e desinfecção das instalações, separar animais por faixa etária, não introduzir animais de outras propriedades antes de serem vermifugados e quarentena, evitar a superlotação das pastagens, rotação de espécies animais e/ou vazio sanitário para quebra do ciclo do parasito nas pastagens e instalações (VIEIRA, 2007).

O uso da fitoterapia é uma alternativa para evitar a resistência, porém há falta de estudos específicos para ovinos que comprovem sua eficácia (COELHO et al. 2017). A Homeopatia é opção viável e recomendada no controle de parasitoses e outras afecções, com redução significativa do número de larvas e melhor ganho de peso (VIEIRA, 2007).

Os animais incapazes de enfrentar um desafio parasitário serão alvos de atenção especial, devendo ser descartados do rebanho, quando identificados ou tratados repetidamente (VIEIRA, 2007), em hipótese alguma os animais sensíveis devem se reproduzir em um rebanho.

\section{Conclusão}

H. contortus deve ser considerada a espécie de maior relevância quando se trata de parasitos gastrointestinais em ovinos. Dessa forma, pode-se afirmar que é o helminto mais prevalente nos rebanhos brasileiros apresentando maior grau de patogenicidade em relação a outros helmintos gastrointestinais, pois gera perdas econômicas significativas. $\mathrm{O}$ controle de parasitoses gastrointestinais possibilita buscar um mercado que prioriza a saúde, bem-estar animal e sustentabilidade, reduzindo concentrações de drogas no ambiente e, consequentemente, melhora da qualidade de carne e leite e buscar a certificação e rastreabilidade para ovinos.

\section{Referências}

AHID, S.M.M. et al. Parasitos gastrintestinais em caprinos e ovinos da região oeste do Rio Grande do Norte, Brasil. Cienc. Anim. Bras, v.9, n.1, p.212-218, 2008.

AMADUCCI, A.G. et al. Parâmetros sanguíneos e OPG (ovos por grama de fezes) de ovelhas mestiças da raça Dorper em diferentes graus do método Famacha. Arq. Ciênc. Vet. Zool. UNIPAR, v.19, n.4, 2016. doi: 10.25110/arqvet.v19i4.2016.6100.

BAKER, K.E. et al. Efficacy of monepantel and anthelmintic combinations against multiple-resistant Haemonchus contortus in sheep, including characterisation of the nematode isolate. Vet. Parasitol. v.186, n.3/4, p.513-517, 2012. doi: 10.1016/j. vetpar.2011.11.060

BATH, G. F.; VAN WYK, J.A. Using the FAMACHA@ system on commercial sheep farms in South Africa. In: PROCEEDINGS OF THE INTERNATIONAL SHEEP VETERINARY CONGRESS. 2001. p. 22-25.

BRAGA, R.M.; GIRARDI, J.L. População de larvas de helmintos infestantes de ovinos em pastagem nativa de Roraima. Pesq. Agropecu. Bras,. v.26, n.4, p.569-574, 1991.

BRITO, D.R.B. et al. Parasitos gastrintestinais em caprinos e ovinos da microrregião do Alto Mearim e Grajaú, estado do Maranhão. Cienc. Anim. Bras., v.10, n.3, p.967-974, 2009.

CEZAR, A.S.; CATTO, J.B.; BIANCHIN, I. Controle alternativo de nematódeos gastrintestinais dos ruminantes: atualidade e perspectivas. Ciênc. Rural, v.38, n.7, p.2083-2091, 2008. doi: 


\section{$10.1590 / \mathrm{S} 0103-84782008000700048$}

CHAGAS, A.C.A.S. et al. Haemonchus contortus: a multipleresistant Brazilian isolate and the costs for its characterization and maintenance for research use. Parasitol. Int., v.62, n.1, p.1-6, 2013. doi: 10.1016/j.parint.2012.07.001

CLIMENI, B.S.O. et al. Hemoncose ovina. Rev. Ciênc. Ele. Med. Vet., v.6, n.11, p.1-7, 2008.

COELHO, M.D.G. et al. Avaliação do uso de extratos vegetais para controle da hemoncos e em ovinos naturalmente infectados. Rev. Ambient. Água, v.12, n.2, p.331-339, 2017. doi: 10.4136/ambi-agua.2020.

COSTA, V.N.M.; SIMOES, A.V.D.; RIET-CORREA, F. Controle das parasitoses gastrointestinais em ovinos e caprinos na região semiárida do Nordeste do Brasil. Pesq. Vet. Bras. v. 31, n. 1, 2011. doi: 10.1590/S0100-736X2011000100010

DA SILVA, D.G. et al. Eficácia anti-helmíntica comparativa entre diferentes princípios ativos em ovinos jovens. PUBVET, v.11, p.313-423, 2017. doi: 1022256/pubvet.v11n4.356-362.

DE ALBUQUERQUE, A.C.A. et al. Development of Haemonchus contortus resistance in sheep under suppressive or targeted selective treatment with monepantel. Vet. Parasitol., v.246, p.112-117, 2017. doi: 10.1016/j.vetpar.2017.09.010.

DO AMARANTE, A.F.T. Controle de verminose ovina. Rev. Conselho Fed. Med. Vet., n.34, p.19-30, 2005.

DO AMARANTE, A.F.T.; RAGOZO, A.M.A.; DA SILVA, B.F. Os parasitas de ovinos. São Paulo: UNESP, 2014

EMERY, D.L.; HUNT, P.W.; LE JAMBRE, L.F. Haemonchus contortus: the then and now, and where to from here? Int. J. Parasitol., v.46, n.12, p.755-769, 2016. doi: 10.1016/j. ijpara.2016.07.001.

ENDO, V.T. et al. Prevalência dos helmintos Haemonchus contortus e Oesophagostomum columbianum em pequenos ruminantes atendidos no setor de Anatomia PatológicaUEM. Rev. Ciênc. Vet. Saúde Públ., v.1, n.2, p.112-118, 2014. doi: $10.4025 /$ revcivet.v1i2.25397

FÁVERO, F.C. et al. Experimental infection of calves with Haemonchus placei and Haemonchus contortus: assessment of parasitological parameters. Vet. Parasitol., v.217, p.25-28, 2016. doi: 10.1016/j.vetpar.2015.12.017.

FERNANDES, M.A.M. et al. Método FAMACHA para detectar anemia clínica causada por Haemonchus contortus em cordeiros lactentes e ovelhas em lactação. Pesq. Vet. Bras., v.35, n.6, p.525530, 2015. doi: 10.1590/S0100-736X2015000600006

FONSECA, Z.S. et al. Relação sexual do parasitismo por Haemonchus contortus em Caprinos (Capra hircus). Embrapa Caprinos e Ovinos, 2011.

GIRÃO, E.S. et al. Prevalência e variação estacional de helmintos gastrintestinais de caprinos no município de Valença do Piaui. Embrapa Meio-Norte-Séries anteriores (INFOTECA-E), 1980.

GONÇALVES, P. C.; VIEIRA, J. M. S. Primeira contribuição à sobrevivência de ovos e larvas de nematódeos de ovinos na pastagem, no Rio Grande do Sul. Rev. Fac. Agron. Vet., v.6, p.95103, 1963.

IBGE - Instituto Brasileiro de Geografia e Estatística. Efetivos dos Rebanhos (Cabeças). 2018.

KAMINSKY, R. et al. Differences in efficacy of monepantel, derquantel and abamectin against multi-resistant nematodes of sheep. Parasitol. Res., v.109, n.1, p.19-23, 2011. doi: 10.1007/ s00436-010-2216-0.
LOPES, B. et al. Levantamento de parasitas gastrointestinais em ovinos sob lotação contínua. Syner. Scye., v.11, p.43-46, 2016.

MARTINS, A.C et al. Haemonchus contortus resistance to monepantel in sheep: fecal egg count reduction tests and randomized controlled trials. Semina: Ciênc. Agrár., v.38, n.1, p.231-238, 2017. doi: 0.5433/1679-0359.2017v38n1p231.

MEDEROS, A.E.; RAMOS, Z.; BANCHERO, G.E. First report of monepantel Haemonchus contortus resistance on sheep farms in Uruguay. Parasit. Vectors., v.7, n.1, p.598, 2014. doi: 10.1186/ s13071-014-0598-z.

MELO, A.C.F.L. et al. Nematódeos resistentes a anti-helmíntico em rebanhos de ovinos e caprinos do estado do Ceará, Brasil. Cienc. Rural, v.33, n.2, p.339-344, 2003.

MILLER, J.E.; HOROHOV, D.W. Immunological aspects of nematode parasite control in sheep. J. Anim. Sci., v.84, n.13, p.E124-E132, 2006. doi: 10.2527/2006.8413_supplE124x.

MOLENTO, M.B.; DANTAS, J.C. Validação do guia Famacha para diagnóstico clínico de parasitoses em pequenos ruminantes no Brasil: resultados preliminares. Anais. Botucatu: Universidade Estadual de São Paulo, 2001.

MONTEIRO, J.P. et al. Acurácia do método FAMACHA no controle seletivo das helmintoses gastrintestinas de caprinos e ovinos. In: ENCONTRO DE INICIAÇÃO CIENTÍFICA DA EMBRAPA CAPRINOS E OVINOS, 6., 2017, Sobral. Anais... Sobral: Embrapa Caprinos e Ovinos, 2017. p.58-59., 2017.

MORAES, F.R.; ROSSI JUNIOR, P.; THOMAZ-SOCCOL, V. Suscetibilidade de ovinos das raças Suffolk e Santa Inês à infecção natural por tricostrongilídeos. Arch. Vet. Sci. v.6, n.2, p.63-69, 2000.

NIETO, L.M. et al. Observações epidemiológicas de helmintos gastrintestinais em ovelhas mestiças manejadas em pastagens com diferentes hábitos de crescimento. Cienc. Anim. Bras., v.4, n.1, p.45-51, 2003.

RADOSTITIS O.M.; BLOOD D.C.; GAY C.C. Clínica veterinária: um tratado de doenças dos bovinos, ovinos, suínos, caprinos e equinos. Rio de Janeiro: Guanabara, 2002.

RAMOS, C.I. et al. Epidemiologia das helmintoses gastrintestinais de ovinos no planalto catarinense. Cienc. Rural., v.34, n.6, p.1889-1895, 2004.

REIS, I.F. Controle de nematoides gastrointestinais em pequenos ruminantes: método estratégico versus Famacha $\odot$. Fortaleza: Universidade Estadual do Ceará, 2004.

ROBERTO, F.F.S. Avaliação de ovinos naturalmente infectados por nematoides gastrintestinais sob pastejo intermitente em cultivares de Brachiaria brizantha. Natal: Universidade Federal do Rio Grande do Norte, 2018.

SALES, N.; LOVE, S. Resistance of Haemonchus sp. to monepantel and reduced efficacy of a derquantel / abamectin combination confirmed in sheep in NSW, Australia. Vet. Parasitol., v.228, p.193-196, 2016. doi: 0.1016/j.vetpar.2016.08.016.

SCZESNY-MORAES, E.A. et al. Resistência anti-helmíntica de nematoides gastrintestinais em ovinos, Mato Grosso do Sul. Pesq. Vet. Bras., v.30, n.3, p.229-236, 2010. doi: 10.1590/S0100736X2010000300007.

SOTOMAIOR, C.S.; THOMAZ-SOCCOL, V. Infecção parasitária em ovinos criados em sistema intensivo: acompanhamento de evolução do parasitismo durante um ano. Hora. Vet., v.20, n.119, p.10-15, 2001.

SPRENGER, L.K. et al. Eficácia do fosfato de levamisol em nematódeos gastrintestinais de caprinos e ovinos. Arch. Vet. Sci., 
v.18, n.1, 2012. doi: 10.5380/avs.v18i1.29393.

STEAR, M.J.; MURRAY, M. Genetic resistance to parasitic disease: particulary of resistance in ruminants to gastrointestinal nematodes. Vet. Parasitol., v.54, p.161-176, 1994. doi: 10.1016/0304-4017(94)90089-2.

STORILLO, V.M. Resistência, resiliência e sensibilidade de ovinos ao Haemonchus contortus: comparações hematológicas e bioquímicas. São Paulo: Universidade de São Paulo, 2016.

TORRES-ACOSTA, J.F.J.; HOSTE, H. Alternative or improved methods to limit gastrointestinal parasitism in grazing sheep and goats. Small. Rum. Res., v.77, p.159-173, 2008. doi: 10.1016/j. smallrumres.2008.03.009.

VAN DEN BROM, R. et al. Haemonchus contortus resistance to monepantel in sheep. Vet. Parasitol., v.209, n.3/4, p.278-280, 2015. doi: 10.1016/j.vetpar.2015.02.026.

VELHO, J. P. et al. Composição bromatológica de silagens de milho produzidas com diferentes densidades de compactação. Rev. Bras. Zootec., v.36, n.5, p.1532-1538, 2007.

VIEIRA, J.P. Métodos alternativos de controle de nematoides gastrintestinais em caprinos e ovinos. SIMPÓSIO INTERNACIONAL SOBRE CAPRINOS E OVINOS DE CORTE. Paraíba, 2007. 